\title{
L'ÉTAT-AKP ET LE GOUVERNEMENT PAR LA CRISE
}

Gilles Dorronsoro

\author{
La Découverte | « Mouvements »
}

$2017 / 2 n^{\circ} 90 \mid$ pages 11 à 18

ISSN 1291-6412

ISBN 9782707197191

Article disponible en ligne à l'adresse :

https://www.cairn.info/revue-mouvements-2017-2-page-11.htm

\section{Pour citer cet article :}

Gilles Dorronsoro, «L'État-AKP et le gouvernement par la crise », Mouvements 2017/2 (n 90), p. 11-18.

DOI 10.3917/mouv.090.0011

Distribution électronique Cairn.info pour La Découverte.

(C) La Découverte. Tous droits réservés pour tous pays.

La reproduction ou représentation de cet article, notamment par photocopie, n'est autorisée que dans les limites des conditions générales d'utilisation du site ou, le cas échéant, des conditions générales de la licence souscrite par votre établissement. Toute autre reproduction ou représentation, en tout ou partie, sous quelque forme et de quelque manière que ce soit, est interdite sauf accord préalable et écrit de l'éditeur, en dehors des cas prévus par la législation en vigueur en France. Il est précisé que son stockage dans une base de données est également interdit. 


\section{LE CONTEXTE : HISTOIRE ET INSTITUTIONS}




\section{L'État-AKP et le gouvernement par la crise ${ }^{1}$}

\section{Par Gilles DORRONSORO*} La monopolisation des ressources économiques et institutionnelles
par le parti au pouvoir caractérise l'État turc depuis sa création.
Cet accaparement systématique explique la difficulté d'une
alternance politique régulière.
1. Je remercie Élise Massicard pour son attentive relecture et ses suggestions.

2. Seule l'expérience du Parti de la mère patrie (Anavatan Partisi, ANAP) semble contredire cette observation, puisqu'il perd les élections du 20 octobre 1991 après huit années passées seul au gouvernement. Mais, au lendemain du coup d'État de 1980, l'armée dispose d'une position dominante dans le jeu politique et dans l'État, l'ANAP a donc une autonomie limitée.

* Professeur de science politique, Paris 1.
]

9 espoir de voir la Turquie devenir une société ouverte et démocratique doit être oublié au moins pour les prochaines années, et la période de libéralisation qui a suivi l'arrivée de l'AKP au pouvoir en 2002 fait de plus en plus figure de parenthèse dans l'histoire politique turque. À son arrivée au pouvoir, le gouvernement AKP (Adalet ve Kalkinma Partisi, Parti de la justice et du développement) - encore fragile - avait fait du projet européen et, plus largement, de l'ouverture démocratique une nécessité pour contrer la menace de coup d'État militaire. Aujourd'hui, l'autoritarisme croissant de l'AKP fait écho à la fois aux pratiques présentes dès le passage au multipartisme (dans les années 1950) et à la multiplication des régimes autoritaires/ nationalistes sur la scène internationale en Russie, en Hongrie et ailleurs. Plus qu'un problème de compatibilité entre l'islam et la démocratie, la situation de la Turquie amène à s'interroger sur l'absence de consensus des élites politiques, la colonisation de l'État par un parti dominant et la manipulation des crises comme instrument de gouvernement.

\section{- Une démocratie sans alternance ?}

Depuis son passage au multipartisme en 1945, et malgré plusieurs décennies de concurrence électorale généralement libre, la Turquie n’a jamais connu d'alternance politique régulière après un gouvernement monopartisan ${ }^{2}$. Depuis 1950 et la première alternance électorale, chaque fois qu'un parti a pu former seul un gouvernement majoritaire, il a réussi à remporter les élections suivantes et à se maintenir sans subir l'usure du pouvoir caractéristique ailleurs. De plus, tous les partis dominants sont issus du courant conservateur, avec des références religieuses en contradiction avec le projet kémaliste initial. C'est le cas du Parti démocrate 
(Demokrat Partisi, DP), qui forme un premier gouvernement en 1950 et remporte à nouveau les élections législatives de 1954 et de 1957, du Parti de la justice (Adalet Partisi, AP) entre 1965 et 1969 et de l'AKP, qui a remporté en 2011 sa troisième élection législative consécutive avec 49,9\% des suffrages, soit le meilleur score électoral de son histoire.

Seuls les coups d'État $(1960,1971)$ permettent à l'armée de mettre fin à cette consolidation d'un parti au pouvoir, sans d'ailleurs pouvoir imposer un parti aux élections. Le coup d'État du 27 mai 1960 met ainsi un terme à dix ans de pouvoir Démocrate, celui du 12 mars 1971 oblige le gouvernement AP de Süleyman Demirel à démissionner au terme de plus de cinq années passées au gouvernement. Enfin, le mémorandum du 28 février 1997, où l'armée exige la dissolution du gouvernement, vise à briser l'élan du Refah, le parti islamiste membre de la coalition au pouvoir, dont les succès électoraux laissaient envisager à terme un gouvernement monopartisan. L'armée s'est ouverte-

La période actuelle est unique, avec la consolidation au pouvoir d'un parti dominant sans possibilité de renversement du gouvernement par une crise institutionnelle.

\section{ment opposée au gouvernement}

AKP formé après les élections de 2002, notamment au moment de l'élection d'un président du parti dominant (2007) ; différents projets de coups d'État ont ensuite été dévoilés, aboutissant à la marginalisation de l'institution militaire dans le champ politique. La réalité et l'ampleur de ces projets de coup d'État restent d'ailleurs discutées, les enquêtes s'étant révélées très irrégulières, mais l'armée n'a au final pas été capable de stopper l'AKP. La période actuelle est donc unique, avec la consolidation au pouvoir d'un parti dominant sans possibilité de renversement du gouvernement par une crise institutionnelle (voire un coup d'État).

\section{- La formation d'un État-parti}

Cette énigme politique, l'absence d'usure des gouvernements monopartisans, s'explique par la formation d'un État-parti qui accumule les ressources au point d'empêcher l'alternance ${ }^{3}$. Il ne s'agit pas d'un retour au parti unique sur le modèle du CHP kémaliste de l'entre-deux guerres, car pour des raisons qui tiennent notamment aux contraintes internationales (appartenance à l'OTAN, candidature à l'UE), le régime AKP maintient une façade démocratique avec des élections concurrentielles. Trois éléments caractérisent ce système comparable, par certains aspects, au PRI (Parti Révolutionnaire Institutionnel) du Mexique ou au gouvernement de la Russie actuelle ${ }^{4}$.

D'abord, le parti dominant-l'AKP - investit l'État en recrutant ses militant.e.s au sein des institutions publiques, afin d'instrumentaliser celles-ci au service du parti. En ce sens, l'installation de l'État-AKP rappelle la décennie 1950, quand le Parti Démocrate a glissé vers l'autoritarisme. Le caractère massif de la politisation des institutions, qui

\footnotetext{
3. Nous reprenons ici des éléments d'un travail effectué avec B. Gourisse, "L'armée turque en politique. Autonomie institutionnelle, formation de coalitions sociales et production des crises ", Revue Française de Science Politique 2015/4, Vol. 65 et "Une clé de lecture du politique en Turquie: les rapports État-Partis ", Politix, $2014 / 3 n^{\circ} 107$.
4. C. Fauconnier, "À quoi sert Russie unie ? Conservatisme et modernisation dans les du parti du pouvoir ", Critique internationale, 4/2011 (n०53), discours de légitimation p. $145-165$.
} 
5. Le système des dépouilles (spoils system) est le principe selon lequel un nouveau gouvernement, devant pouvoir compter sur la loyauté partisane de ses fonctionnaires, remplace celles et ceux qui sont en place par des fidèles.

6. E. Massicard, "Une décennie de pouvoir AKP en Turquie : vers une reconfiguration des modes de gouvernement?", Les études du CERI, $\mathrm{n}^{\circ} 205$, juillet 2014 .

7. I. ERdinç, "Répression syndicale en Turquie sous le gouvernement AKP : vers une perte d'autonomie des syndicats?" à paraître dans Travail et emploi et son article dans ce dossier ; D. YANKAYA, La nouvelle bourgeoisie islamique. Le modèle turc, Paris, PUF, coll. "Proche-Orient ", 2013. va bien au-delà du spoil system ${ }^{5}$ à la manière des États-Unis, transforme la nature même des institutions, dont la neutralité est mise en cause. La nomination des premiers gouvernements AKP a vu en particulier s'éroder l'indépendance de la branche judiciaire, phénomène qui s'est radicalement accéléré avec les purges qui ont suivi le coup d'État manqué de juillet 2016. Bel exemple de l'ambiguité de l'AKP, la réforme de la constitution de 2011 avait été reçue à l'époque comme garantissant une plus grande autonomie de la branche judiciaire, alors qu'elle annonçait sa mise au pas. La fusion de l'État et du parti permet la monopolisation des ressources étatiques, utilisées dans la construction d'un clientélisme politique de grande ampleur ${ }^{6}$. La base militante de l'AKP trouve ainsi des avantages concrets au niveau national ou municipal à son adhésion au parti, alors que les milieux d'affaires proches du pouvoir bénéficient des marchés publics. La possibilité pour l'AKP d'être à la fois un parti populaire et un parti pour les élites s'explique ainsi par la captation des ressources institutionnelles.

Ensuite, l'État-parti influe sur la société par une présence directe ou indirecte dans tous les secteurs d'activité, notamment économique, médiatique et syndical, par le biais de relais soit appartenant à la même mouvance islamiste que le pouvoir, soit d'une façon ou d'une autre dépendants de l'État ${ }^{7}$. L'autonomie des différents secteurs d'activité est limitée et on a, tendanciellement, une politisation des sphères de la vie sociale qui profite à l'AKP. On retrouve ici la structure complexe du mouvement islamiste et ses différentes activités : fondations charitables, syndicat (Hak-İş), associations culturelles, syndicats patronaux (MÜSİAD), médias islamistes/conservateurs qui soutiennent le gouvernement.

Enfin, le pouvoir intervient directement contre celles et ceux qui critiquent le régime, notamment des journalistes, des syndicalistes, des militant e.s de gauche, des universitaires, des juges. La multiplication des procédures judiciaires et les pressions administratives sont l'arsenal quotidiennement utilisé pour assurer la marginalisation des discours critiques. Par exemple, la plupart des médias nationaux sont désormais soit liés au pouvoir, soit dépendants de lui. En mars 2017, le pouvoir a obtenu sans difficulté la démission du rédacteur en chef de l'un des principaux journaux turcs, Hürriyet, pourtant progouvernemental, à la suite d'un article modérément critique sur les rapports entre le gouvernement et l'armée. De même, la reprise en main des universités s'est faite notamment par la nomination systématique de recteur'e.s lié.e.s au pouvoir. De plus, pour les milliers d'universitaires contre lesquels des procédures judiciaires ont été engagées, toute expression publique devient dangereuse. L'incertitude juridique qui pèse sur les individus a pour objectif de paralyser leur engagement professionnel ou politique. 
L'ampleur de la répression est telle que, pour la première fois depuis le coup d'État du 12 septembre, des intellectuel-le-s, des journalistes et des militant·e.s sont en exil en Europe. Certain·e.s diplomates ont également demandé l'asile politique, environ 136 à l'Allemagne depuis juillet $2016^{8}$.

\section{- Le gouvernement par la crise}

L'État-AKP, avec sa base militante et ses relais dans différents champs sociaux, s'est mis en position de résister à la fois aux mobilisations contestataires, aux crises institutionnelles et à la compétition électorale. En réalité, la création/exploitation des crises est devenue une ressource essentielle pour avancer l'agenda du pouvoir et marginaliser l'opposition. Ce répertoire n'est pas une invention de l'AKP, et l'armée a systématiquement exploité ce registre, mais son caractère systématique - surtout pour un gouvernement civil - marque une rupture. Ainsi, le coup d'État raté du 15 juillet 2016, les attentats de l'État islamique, la guérilla kurde sont autant d'occasions d'installer un état d'urgence localisé ou national qui affranchit le pouvoir des règles du droit.

Lors du coup d'État raté de juillet 2016, on a pu voir l'ampleur des ressources dont dispose le pouvoir. D'une part, l'AKP est le seul parti à disposer d'une base nationale aussi forte et homogène, et les militant.e's AKP sont immédiatement descendu·e·s dans la rue pour faire échec aux putschistes, ce qui représente une première dans l'histoire des coups d'État militaires en Turquie. D'autre part, aucune institution non militaire n'a rallié les putschistes ; les mosquées (qui dépendent de la présidence des Affaires religieuses) ont même appelé à la résistance dans tout le pays. En réalité, la tentative de coup d'État a d'abord mis en évidence l'affaiblissement progressif de l'armée depuis 2002 et son incapacité à construire une coalition politique et sociale comme en 1980. Les putschistes ont été incapables de mobiliser les secteurs sécuritaires (armée et police) notamment du fait que la hiérarchie militaire n'a pas été impliquée (comme lors du coup d'État de 1960). En réalité, le coup d'État semble avoir été mal préparé, et représente surtout une réaction devant les menaces de purges qui s'étaient multipliées durant les années précédentes.

Le coup d'État raté de 2016 a également été l'occasion de purger l'administration via des décrets ayant force de loi dans le cadre de l'état d'urgence. Environ 100000 personnes ont été renvoyées et des centaines d'institutions ont été fermées (dont des journaux) ; l'enseignement, la magistrature et la police ont été les secteurs les plus touchés. Si les partisan'e·s de Fethullah Gülen ont certes été visé·e·s pour leur
8. http://www. faz.net/aktuell/ politik/ausland/136tuerkische-diplomatensuchen-asyl-indeutschland-14893476. html

\section{L'État-AKP, avec sa base militante et ses relais dans différents champs sociaux, s'est mis en position de résister à la fois aux mobilisations contestataires, aux crises institutionnelles et à la compétition électorale.}


9. Sur ces mobilisations, voir J.-F. PÉrouse, " Le parc Gezi : dessous d'une transformation très politique ", Métropolitiques, 24 juin 2013. URL : http://www. metropolitiques.eu/ Le-parc-Gezi-dessousd-une.html et B.

Montabone, "Droit à la ville et contestation de l'ordre moral urbain en Turquie ", EchoGéo, Sur le Vif, en ligne depuis le 10 Octobre 2013. URL : http://echogeo.revues. org/13567 ; DOI : 10.4000/echogeo. 13567
10. Les représentations culturelles de la classe dirigeante, c'est-àdire kémaliste pour la Turquie républicaine, se sont mal diffusées dans la société. Les raisons en sont complexes, mais on notera que le kémalisme a plutôt joué de la distance culturelle pour imposer le pouvoir d'une élite numériquement limitée. La " modernisation " kémaliste est plus l'affirmation d'une fermeture sociale qui protège l'entre-soi des élites qu'un projet émancipateur. implication supposée dans le coup d'État, la purge s'est généralisée pour atteindre l'ensemble des critiques du régime, notamment les journalistes, les juges et les enseignant $\cdot$ es. Ces licenciements donnent en outre la possibilité au gouvernement de recruter des militant·e's ou des proches de l'AKP dans des secteurs stratégiques, notamment la magistrature et les institutions de sécurité.

Par ailleurs, les protestations de Gezi en 2013 ont montré la difficulté qu'il y a à étendre une mobilisation au-delà de son public initial pour en faire un instrument de contestation plus global du gouvernement en place'. L'hostilité des médias explique, au moins en partie, l'échec de Gezi, mais c'est surtout cette incapacité de la mobilisation à s'étendre (en dehors de quelques exceptions) qui doit attirer notre attention. Les partis, les médias, les syndicats n'ont que peu ou pas soutenu le mouvement. Les réseaux sociaux peuvent jusqu'à un certain point servir d'alternative aux médias, ce qui explique probablement les différentes mesures prises ces dernières années pour bloquer ponctuellement certains réseaux et, surtout, engager des procédures judiciaires contre les internautes suite à des propos critiques du pouvoir.

Enfin, l'élection de juin 2015 montre la capacité du pouvoir à maîtriser le jeu électoral. Le gouvernement, suite notamment au bon résultat du mouvement kurdiste HDP (Halklarm Demokratik Partisi, Parti démocratique des peuples, qui a mobilisé au-delà des Kurdes), n'avait pas été en mesure de former un gouvernement seul. Plutôt que de former une coalition, Recep Tayyip Erdoğan a préféré une stratégie de pourrissement, refusant de former un gouvernement et provoquant une forte montée de la violence dans les provinces kurdes. En conséquence, les élections de novembre 2015 ont vu un ralliement autour de l'AKP (qui les a remportées) tandis que le MHP (Milliyetçi Hareket Partisi, Parti du mouvement nationaliste, extrêmedroite) passait de $16,45 \%$ à 11,9\%, et le HDP de $12,96 \%$ à 10,76\%.

\section{- Hégémonie et conflits identitaires}

Depuis la fondation de la république de Turquie, aucun pouvoir n'a pu bénéficier d'une hégémonie au sens gramscien du terme ${ }^{10}$. L'absence de consensus des élites politiques sur la nature du régime a conduit de façon circulaire à une appropriation de l'État qui interdit l'alternance. En ce sens, le mouvement islamiste a été victime de nombreuses interdictions et le comportement actuel de l'AKP reflète à la fois une arrogance par rapport aux autres partis et aux institutions, mais aussi une forme d'insécurité. L’opposition, même parfaitement légaliste, devient illégitime, ce qui rappelle les périodes où l'armée dominait le champ politique après le coup d'État de 1980.

Cette construction du politique, où l'opposition est rapidement stigmatisée comme un ennemi intérieur, voit ses effets renforcés par la manipulation des clivages identitaires. Le kémalisme originel des années 1920 est marqué par une construction identitaire très particulière, qui voit la négation de certains groupes ethniques, en particulier les Kurdes. Il faut attendre les années 1990 pour voir la situation changer avec la reconnais- 
sance des Kurdes, l'autorisation d'utiliser la langue kurde progressivement accordée, etc. Cette apparente libéralisation avec la multiplication de discours (livres, chansons...) sur les Kurdes et en kurde ne doit pas dissimuler le fait que la hiérarchie ethnique qui renvoie les Kurdes à des différences essentielles, qui reprend les formes classiques de l'orientalisme, est restée essentiellement en place ${ }^{11}$. En fait, les revendications culturelles n'ont pas réglé la question et une forte minorité de Kurdes votent pour les partis kurdistes et considèrent le PKK comme un acteur légitime. Le retour à la lutte armée après des tentatives ratées de négociation a eu pour conséquence une radicalisation qui au final profite au régime. Le PKK a lancé la lutte armée dans les villes, ce qui a été un désastre à la fois pour la population et pour le mouvement kurde légal. Profitant de l'état de guerre, le gouvernement a entrepris un démantèlement systématique des institutions contrôlées par le mouvement kurde, à commencer par les municipalités qui représentaient la base du pouvoir du mouvement depuis les années 1990. La polarisation politique sur fond d'affrontements militaires a pour effet le démantèlement systématique du mouvement kurde sous l'effet d'une répression sans équivalent depuis les années 1990.

Par ailleurs, on voit s'exacerber une autre fracture de la société turque, qui touche à la reconnaissance des alévi·es's comme groupe distinct. Les gouvernements turcs ont toujours refusé de reconnaître l'alévisme comme une religion et les pressions à la conversion au sunnisme (circoncision, construction de mosquées) ont souvent été fortes, par exemple lors du service militaire. Après son arrivée au pouvoir, l'AKP a lancé une politique d'ouverture aux alévi·e·s entre 2007 et 2009 environ. Depuis la fin des années 2000, le gouvernement a progressivement affirmé le caractère sunnite du pouvoir, notamment par le biais de sa politique régionale, et les alévi·e·s ont été de fait écarté·e·s du pouvoir (elles et ils sont en nombre négligeable à l'AKP) et des postes à responsabilité au sein des institutions d'État. Les mobilisations de Gezi ont, à tort ou à raison, été interprétées comme dominées par les alévi·e.s et par les partis d'extrême-gauche, d'autant que le leader du principal parti d'opposition est un alévi. Les mobilisations proErdoğan lancées par des sunnites libanais témoignent par exemple de la popularité réelle du dirigeant turc au Moyen-Orient, chez certain·e·s sunnites confrontée-e's à des régimes répressifs dominés par des non sunnites (en Syrie et en Irak). Cette exclusion désormais actée d'une minorité a notamment pour effet de constituer des élites au pouvoir homogènes, dont les discours et les politiques ne sont pas modérés par des enjeux électoraux, dans la mesure où la base sunnite (hors Kurdes) devient le seul enjeu.
11. C. SCALBERT-YüCEL, "Diversité culturelle et hiérarchie ethnique. L'usage des catégories dans le conflit kurde en Turquie ", in

G. Dorronsoro ET

O. Grojean (eds) Identités et politique. De la différenciation culturelle au conflit, Presses de Science Po, Paris, 2015, 117-144.

\section{Cette construction du politique, où l'opposition est rapidement stigmatisée comme un ennemi intérieur, voit ses effets renforcés par la manipulation des clivages identitaires.}




\section{- Conclusion}

La trajectoire du régime turc m'inspire trois conclusions. Premièrement, la domination de l'AKP sur les ressources étatiques et ses relais dans les différents champs sociaux rendent l'alternance de plus en plus difficile à envisager. Les mouvements sociaux n'ont que peu de relais politiques ou syndicaux et courent le risque d'être isolés et délégitimés face au pouvoir. Les institutions marchent désormais au service de l'AKP et les élections, même formellement concurrentielles, jouent largement au désavantage de l'opposition. Par exemple, il n'y a plus de régulation des temps de parole pendant la campagne électorale, ce qui avantage le gouvernement qui domine sans partage les médias. Le référendum d'avril 2017 sur la présidentialisation du régime sera en un sens le dernier test pour

\section{Le référendum d'avril 2017 sur la présidentialisation du régime sera en un sens le dernier test pour la démocratie turque.}

la démocratie turque. Les mauvais résultats économiques de ces derniers mois - faible croissance, effondrement de la lire et inflation à plus de $9 \%$ - et les craintes par rapport au caractère de plus en plus autoritaire du régime expliquent probablement les réticences de l'électorat telles qu'elles sont perceptibles dans les sondages. Si le pouvoir parvient malgré tout à l'emporter, on voit mal comment une alternance pourrait prendre place. Plus fondamentalement, l'AKP ne peut plus accepter de perdre le pouvoir du fait des accusations multiples de corruption et de répression illégale qui rendent une alternance pacifique difficile à envisager. Le destin d'Adnan Menderes, premier ministre entre 1950 et 1960 et pendu à la suite du coup d'État de 1960, est probablement présent à l'esprit d'Erdoğan et des militant·e·s de l'AKP. Au-delà de la manipulation des médias et des réseaux clientélistes, le pouvoir pourrait maintenant être tenté par la fraude pure et simple.

Deuxièmement, au sein même de l'AKP, Erdoğan exerce un pouvoir de plus en plus solitaire, du fait de la marginalisation des personnalités historiques du mouvement comme Gül (ancien président). En ce sens, Erdoğan n'est plus dans une logique d'arbitrage entre des courants à l'intérieur du parti ${ }^{12}$. La présidentialisation en marche du régime va installer un exécutif sans contre-pouvoir législatif ou judiciaire significatif si Erdoğan remporte le référendum d'avril. Seule sa succession pourrait créer une dynamique d'ouverture dans la mesure où l'extrême personnalisation du système est couplée avec une absence d'héritier désigné (dans sa famille ou en dehors).

Troisièmement, le régime a jusque-là tiré avantage de toutes les crises mais, en conséquence, a renforcé les clivages au sein de la société. Ce verrouillage progressif de la société turque confine aux marges du système politique une bonne partie des alévi·e·s et des Kurdes, soit probablement plus du tiers de la population. 